\title{
Air Shower Development, Pion Interactions and Modified EPOS Model
}

\author{
Tanguy Pierog $^{a}$, B.Guiot ${ }^{b}$, and K. Werner ${ }^{b}$ \\ ${ }^{a}$ Karlsruhe Institute of Technology (KIT), IKP, D-76021 Karlsruhe, Germany \\ ${ }^{b}$ SUBATECH, University of Nantes, F-44000 Nantes, France \\ E-mail: tanguy.pierog@kit.edu
}

\begin{abstract}
In detailed air shower simulations, the uncertainty in the prediction of shower observable for different primary particles and energies is currently dominated by differences between hadronic interaction models. With the results of the first run of the LHC, the difference between post-LHC model predictions has been reduced at the same level than experimental uncertainties of cosmic ray experiments. At the same time new type of air shower observables, like the muon production depth, has been measured adding new constraints on hadronic models. Currently no model is able to reproduce consistently all mass composition measurement possible within the Pierre Auger Observatory for instance. Using new modifications in EPOS and LHC data, we will show how air shower measurements can be used to constrain pion-air interactions in kinematic phase space which can not be tested by laboratory experiments. The goal being a model which can reproduce all primary mass composition measurements from air showers in a consistent way.
\end{abstract}

The 34th International Cosmic Ray Conference,

30 July- 6 August, 2015

The Hague, The Netherlands 


\section{Introduction}

Knowing the elemental composition of cosmic ray particles arriving at Earth is of crucial importance to understand the production and propagation of cosmic rays. Unfortunately, cosmic rays can be measured only indirectly above an energy of $10^{14} \mathrm{eV}$ through the cascades of secondary particles, called extensive air-showers (EAS). With the operation of modern large-scale experiments, the reliability of air-shower simulations has become the source of the largest systematic uncertainty in the interpretation of cosmic-ray data due to the large uncertainties in modelling the hadronic interactions driving the EAS development [1].

The Large Hadron Collider (LHC) at the CERN laboratory allows us to access, for the first time, the energy region above the cosmic ray spectral knee in the laboratory. Therefore an analysis of inclusive particle data taken at the LHC is particularly interesting for constraining existing hadronic interaction models and for testing possible new mechanisms of hadron production [2]. There are several hadronic interaction models commonly used to simulate air showers. For recent air shower analysis, the two high energy models which were updated to take into account LHC data at 7 TeV: QgsjetII-03 [3, 4] changed into QgSJetII-04 [5] and Epos 1.99 [6, 7] replaced by EpOs LHC (v3400) [8], are now commonly used. There is no major change in these models but in addition to some technical improvements, some parameters were changed to reproduce TOTEM [9] cross sections and other LHC data. Both are based on Gribov-Regge multiple scattering, perturbative QCD and string fragmentation. Results obtained by these models are now much more consistent than before LHC [10], but the description of EAS data is still not fully consistent.

In this paper, we will discuss changes in the hadronic model predictions after LHC data which leads to a worth description of an air shower observables: the muon production depth (MPD). In the first section, we will explain basic principles of EPOS. We will then describe how the Pierre Auger Observatory [11] measure MPDs and the comparison to post-LHC models. Using fast simulations done with CONEX [12], we will test a possible change of pion interaction in EPOS which could reconcile the measurement of $\left\langle X_{\max }^{\mu}\right\rangle$ with LHC data.

\section{EPOS model}

In this paper we will focus on EPOS LHC which is a minimum bias monte-carlo hadronic generator used for both heavy ion interactions and cosmic ray air shower simulations. It is based on EPOS 1.99 retuned to reproduce LHC data on a higher precision level. As most of high energy hadron-hadron interaction models, it is based on pQCD but associated to the Gribov-Regge Theory [13] and can be seen as an exchange of multiple "parton ladders" between the two hadrons.

In EPOS, the term "parton ladder" is actually meant to contain two parts [13]: the hard one, as discussed above, and a soft one, which is a purely phenomenological object, parametrized in Regge pole fashion. This is the so called Pomeron used as elementary parton-parton interaction in EPOS.

In additions to the parton ladder, there is another source of particle production: the two offshell remnants, see Fig. 1. We showed in ref. [14] that this "three object picture" can solve the "multi-strange baryon problem" of conventional high energy models, see ref. [15]. 


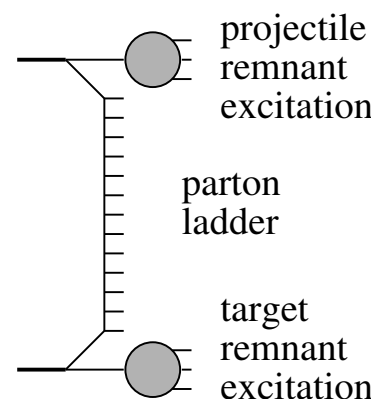

Figure 1: Elementary parton-parton scattering: the hard scattering in the middle is preceded by parton emissions attached to remnants. The remnants are an important source of particle production even at intermediate energies $(\sim 100 \mathrm{GeV} \mathrm{cms})$.

Hence EPOS is a consistent quantum mechanical multiple scattering approach based on partons and strings [13], where cross sections and the particle production are calculated consistently, taking into account energy conservation in both cases (unlike other models where energy conservation is not considered for cross section calculations [16]). The main consequence of this energy sharing process is that the number of Pomerons generated event-by-event does not follow a simple Poissonian distribution. As a consequence it is much less probable to produce events with a very large number of Pomerons (large multiplicity) compared to the standard Gribov-Regge approach like in QGSJETII. Nuclear effects related to Cronin transverse momentum broadening, parton saturation, and screening have been introduced into Epos [6]. Furthermore, high density effects leading to collective behavior in heavy ion collisions are also taken into account [17].

Energy momentum sharing and remnant treatment are the key points of the model concerning air shower simulations because they directly influence the multiplicity and the inelasticity of the model. The remnant mass distribution in case of diffraction, which is a simple power law defined as $\left(\mathrm{M}^{2}\right)^{-\alpha}$ where $\alpha$ is a parameter, is particularly important for the discussion of this paper since it completely drives the particle production in case of diffraction. A higher value of $\alpha$ as in Epos LHC ( $\alpha=1.05)$ compared to EpOs $1.99(\alpha=0.45)$ leads to lower multiplicity and then higher elasticity.

\section{MPD measurements}

It has been shown in [10] how LHC data improve the description of EAS using updated hadronic interaction models. But in fact in one particular case, the update of EPOs leads to inconsistent results: the muon production depth measured by the Pierre Auger Observatory [18].

Muons reaching the ground have a time structure which can be used to obtain the distribution of muon production distances along the shower axis. The basis of the measurement done by the Pierre Auger Observatory is a theoretical framework originally developed in $[19,20]$ and updated in [21]. Using the Gaisser-Hillas function, the point along the shower axis where the production of muons reaches a maximum, $X_{\max }^{\mu}$, can be fitted. Simulations show that the $X_{\max }^{\mu}$ distribution varies as a function of the mass of the primary particle. For heavier primaries, the average value of $X_{\max }^{\mu}$ is smaller and the distribution narrower compared with that for lighter particles [18]. This behavior 
is independent of the energy of the primary cosmic ray. As a consequence this measurement was done first to measure the primary cosmic ray mass.

The evolution of the measured $\left\langle X_{\max }^{\mu}\right\rangle$ as a function of energy is shown in Fig. 2. The proposed method can be used as a tool to investigate the validity of hadronic interaction models. In Fig. 2 we can see how QGSJETII-04 and Epos LHC predict, for both proton and iron, the same muonic elongation rate (rate of evolution of $\left\langle X_{\max }^{\mu}\right\rangle$ with energy) but with considerable differences in the absolute value of $\left\langle X_{\max }^{\mu}\right\rangle$.

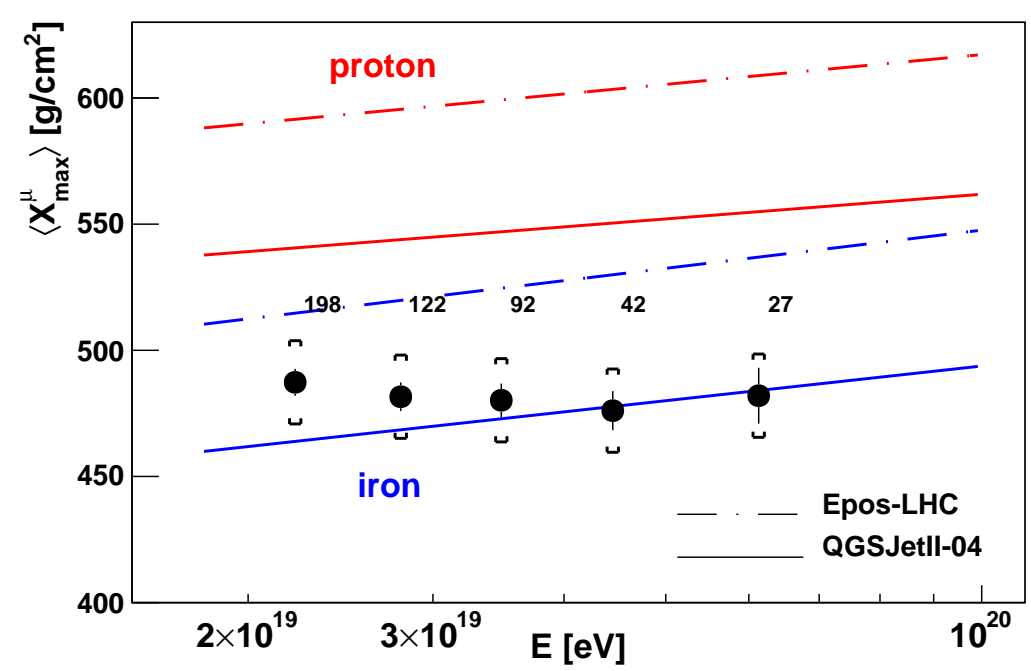

Figure 2: $\left\langle X_{\max }^{\mu}\right\rangle$ as a function of energy from [18]. The predictions of different hadronic models for protons and iron are shown. Numbers indicate the number of events in each energy bin, and brackets represent the systematic uncertainty.

In particular the data are out of the range defined by the proton and iron primary mass when EPOS LHC is used for the simulation while QGSJETII-04 gives consistent results. In previous analysis [22], EPOS 1.99 was giving consistent results, so the important shift observed in MPD simulated with EPOS LHC can easily be identified as a consequence of the change in elasticity due to the corrections in diffractive interactions needed to reproduce the rapidity gap distributions measured by the ATLAS collaboration [23]. We can see on Fig. 3 left-hand side that none of the pre-LHC models could describe the large rapidity gap cross-section, while EPOS LHC gives reasonable results.

\section{MPD and hadronic interactions}

The change of the parameters needed to describe the rapidity gap correctly (the diffractive cross-section and the diffractive mass distribution ( $\alpha$ as explained in Sect. 2 ) affected both proton and pion interactions because the same parameters are used for both types of projectiles. While the change of diffraction and thus of elasticity in proton interaction has very little impact on $\left\langle X_{\max }^{\mu}\right\rangle$, it appears that MPD are extremely sensitive to the elasticity of pion interactions. This can be 

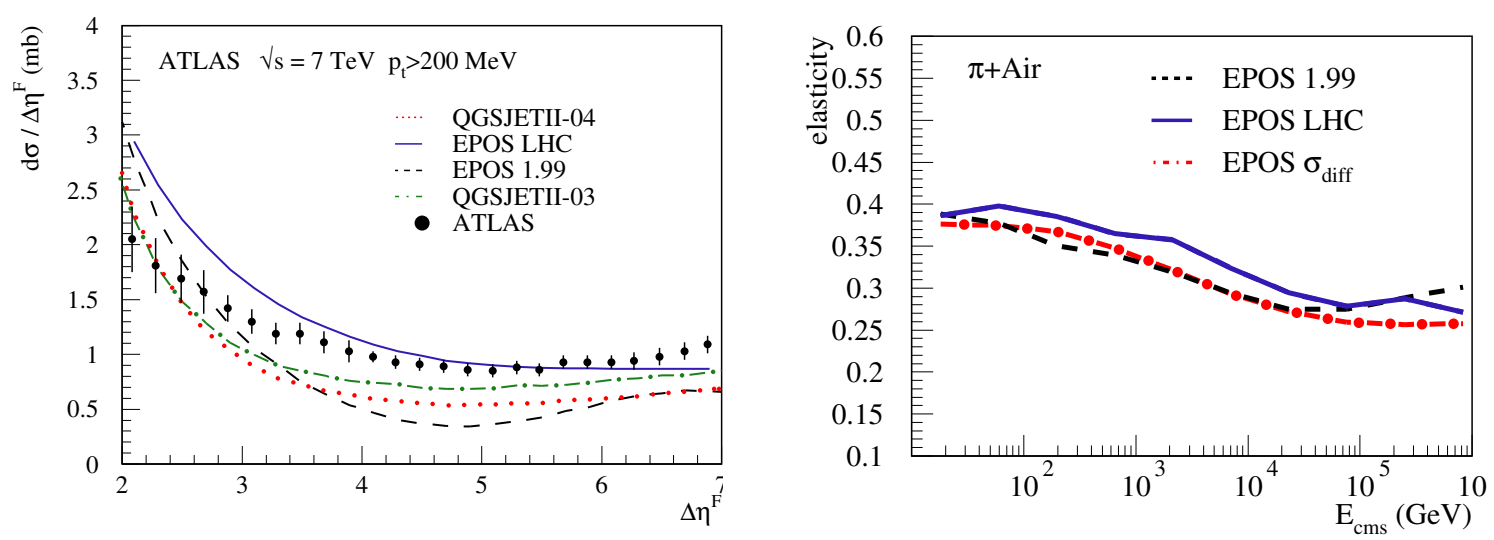

Figure 3: ATLAS measurement of the pseudorapidity gap $\Delta \eta F$ for particles with $p_{t, \text { cut }}>200 \mathrm{MeV}$ in minimum bias events at $7 \mathrm{TeV}$ [23] compared to EpOs LHC (solid line), EpOs 1.99 (dashed line), QGSJETII-04 (dotted line) and QGSJETII-03 (dash-dotted line) simulations.

understood by the fact that muons are produced at the end of the hadronic cascade after many generations of mainly pion-air interactions. As a consequence of this cumulative effect, even a small increase of only about $10 \%$ of the elasticity of pion-air interactions can lead to large shift in $\left\langle X_{\max }^{\mu}\right\rangle$. The elasticity of pion-air interactions in EpOs 1.99 and EpOS LHC are shown on Fig. 3 right-hand side.

To check this hypothesis, the diffractive cross-section for pion interactions has been reduced in EPOS LHC with respect to the inelastic one (which should not be changed) to get a reduction of about $10 \%$ of the elasticity of the pion-air interactions to be similar as the one in EPOS 1.99 as shown on Fig. 3 right-hand side. In the following, we will call this modified version Epos $\sigma_{\text {diff. }}$ Since the diffractive mass distribution is narrower in EPOS LHC with respect to EPOS 1.99 (due to LHC data), to have a similar low elasticity in both EPOS $\sigma_{\text {diff }}$ and EPOS 1.99 , the ratio $\sigma_{\text {diff }} / \sigma_{\text {ine }}$ have to be much smaller in the test version. This is illustrated on Fig. 4 left-hand side where $\sigma_{\text {ine }}, \sigma_{\text {diff }}$ and $\sigma_{\mathrm{SD}}$ are compared for the different EPOS version. In all cases the single diffractive cross-section $\sigma_{\mathrm{SD}}$ is very similar because of the constraint of other data.

As a result $\left\langle X_{\max }^{\mu}\right\rangle$ is reduced by about $30 \mathrm{~g} / \mathrm{cm}^{2}$ and is very similar to the one obtained using Epos 1.99 as shown on Fig. 5 left-hand side. The diffraction has not been changed for proton interactions to keep full compatibility with LHC data. To avoid bias due to electromagnetic shower component, experimental MPDs are built by counting muons at ground in the distance range $1700 \mathrm{~m} \leq \mathrm{r} \leq 4000 \mathrm{~m}$ for inclined events $\left(\theta>\sim 60^{\circ}\right)$. Thus the reconstructed MPD is not the true one as produced by CONEX for instance. Since EPOS LHC $\sigma_{\text {diff }}$ is only implemented in Conex, 3D simulations are not available and thus it is not possible to compare the results with experimental data. That is why we use EPOS 1.99 as reference model which is compatible with data. Further tests have to be done using CORSIKA 3D simulations [26] and experimental reconstruction of the MPDs.

Such a small change is compatible with all pion-nucleus data that are available at low energy and thus EPOS LHC and EPOS $\sigma_{\text {diff }}$ cannot be discriminated by accelerator data as shown on Fig. 4 right-hand side. But the effect on MPD is so strong that data from the Pierre Auger Observatory 

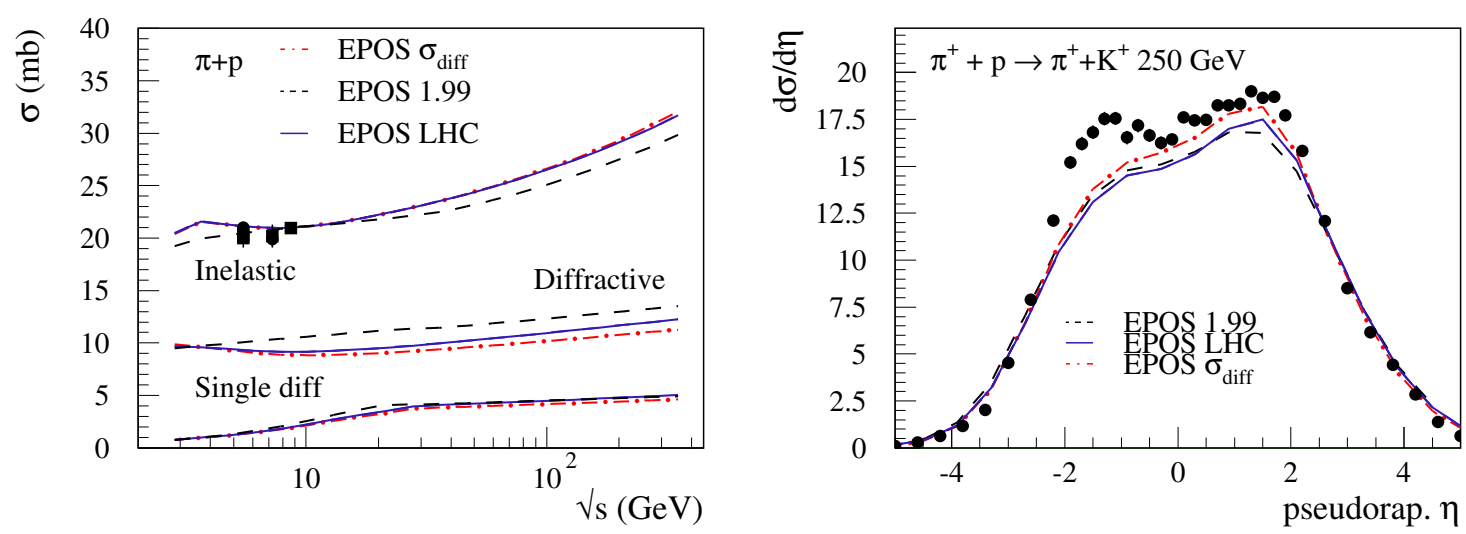

Figure 4: Left-hand side: Inelastic, (all) diffractive and single diffraction cross-section for pionproton interaction as a function of the center-of-mass energy (data from [24]). Right-hand side: pseudorapidity distribution of positively charged particles for pion-proton interaction at $250 \mathrm{GeV}$ lab energy as measured by the NA22 collaboration [25]. Full line is EPOS LHC simulations, dashed line is from EPOS 1.99 and dashed-dotted line is the modified EPOS $\sigma_{\text {diff }}$ version for pion diffraction.
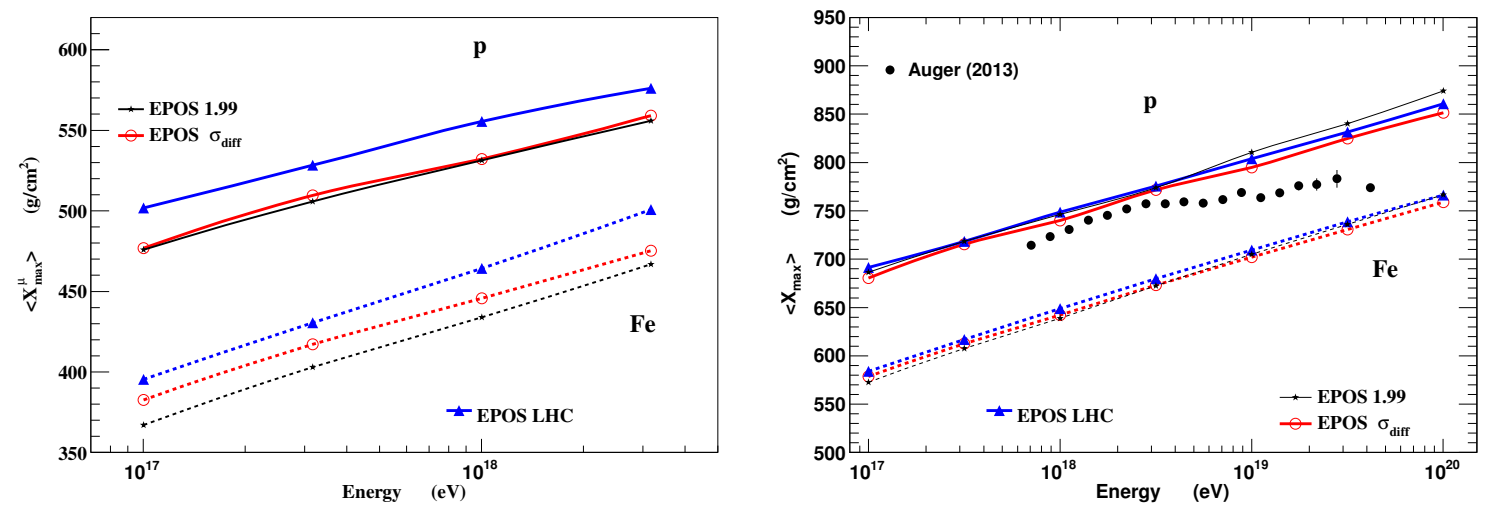

Figure 5: $\left\langle X_{\max }^{\mu}\right\rangle$ (left) and $\left\langle X_{\max }\right\rangle$ (right) as a function of energy for EPOS LHC (triangles), Epos 1.99 (stars) and EPOS $\sigma_{\text {diff }}$ with a reduced elasticity in pion-air interactions (open circles). Data on $\left\langle X_{\max }\right\rangle$ are from the Pierre Auger Observatory [27].

can be used to constrain diffraction in pion interactions to get consistent results between the mean logarithmic mass which can be extracted from $\left\langle X_{\max }^{\mu}\right\rangle$ and the one deduced from $\left\langle X_{\max }\right\rangle$ which has very little dependence on pion hadronic interaction (see Fig. 5 right-hand side where a shift of only about $10 \mathrm{~g} / \mathrm{cm}^{2}$ is observed between EPOS LHC and EPOS $\sigma_{\text {diff }}$ ). Thus we can say that pion diffraction is very important to control the muon production but only indirect measurements from old experiments are available. This leads to a large uncertainty on the muon production rate and probably on the total number of muons which are produced, since a higher inelasticity is linked to a higher number of muons at ground [28]. 


\section{Summary}

Air shower measurements, such as the muon production depth, can also give very strong constrain on hadronic interactions in particular for pion interactions for which cumulative effects due to the hadronic cascade can be observed. For such interactions where a pion is the projectile, LHC data do not provide any direct test since the beam particles are either protons or nuclei. In the case of EPOS LHC it is thus possible to keep the same description of LHC data and at the same time to have a better description of MPD measurement by changing pion-air interactions only.

To conclude, we can say that LHC data contribute a lot to reduce the uncertainties in air shower simulations, providing better tools to analyze cosmic ray data. The differences between the hadronic models have been reduced but one should keep in mind that there are still uncertainties in the models themselves which have to be better quantified and transferred to the calculation of the systematic errors in EAS analysis (pion and kaon projectiles, nuclear target etc...). And consistency of different EAS observables can and should be used to test the hadronic interaction models.

\section{References}

[1] R. Engel, D. Heck, and T. Pierog, Extensive air showers and hadronic interactions at high energy, Ann.Rev.Nucl.Part.Sci. 61 (2011) 467-489.

[2] B. Alessandro, D. Bergman, M. Bongi, A. Bunyatyan, L. Cazon, et al., Hadron-Hadron and Cosmic-Ray Interactions at multi-TeV Energies, arXiv:1101.1852.

[3] S. Ostapchenko, Non-linear screening effects in high energy hadronic interactions, Phys. Rev. D74 (2006) 014026, [hep-ph/0505259].

[4] S. Ostapchenko, On the re-summation of enhanced pomeron diagrams, Phys. Lett. $\mathbf{B 6 3 6}$ (2006) 40-45, [hep-ph/0602139].

[5] S. Ostapchenko, Monte Carlo treatment of hadronic interactions in enhanced Pomeron scheme: I. QGSJET-II model, Phys.Rev. D83 (2011) 014018, [arXiv: 1010 . 1869].

[6] K. Werner, F.-M. Liu, and T. Pierog, Parton ladder splitting and the rapidity dependence of transverse momentum spectra in deuteron gold collisions at rhic, Phys. Rev. C74 (2006) 044902, [hep-ph/0506232].

[7] T. Pierog and K. Werner, EPOS Model and Ultra High Energy Cosmic Rays, Nucl.Phys.Proc.Suppl. 196 (2009) 102-105, [arXiv: 0905.1198 ].

[8] T. Pierog, I. Karpenko, J. Katzy, E. Yatsenko, and K. Werner, EPOS LHC: test of collective hadronization with LHC data, arXiv: 1306.0121.

[9] TOTEM Collaboration Collaboration, T. Csörgö et al., Elastic Scattering and Total Cross-Section in $p+p$ reactions measured by the LHC Experiment TOTEM at $\sqrt{s}=7 \mathrm{TeV}$, Prog.Theor.Phys.Suppl. 193 (2012) 180-183, [arXiv:1204.5689].

[10] T. Pierog, LHC data and extensive air showers, EPJ Web Conf. 52 (2013) 03001.

[11] Pierre Auger Collaboration Collaboration, J. Abraham, Measurement of the Depth of Maximum of Extensive Air Showers above $10^{18}$ eV, Phys. Rev. Lett. 104 (2010) 091101, [arXiv: 1002.0699 ].

[12] T. Bergmann et al., One-dimensional hybrid approach to extensive air shower simulation, Astropart. Phys. 26 (2007) 420-432, [astro-ph / 0606564 ]. 
[13] H. J. Drescher, M. Hladik, S. Ostapchenko, T. Pierog, and K. Werner, Parton-based gribov-regge theory, Phys. Rept. 350 (2001) 93-289, [hep-ph / 0007198 ].

[14] F. Liu, J. Aichelin, M. Bleicher, H. Drescher, S. Ostapchenko, et al., Constraints on models for proton proton scattering from multistrange baryon data, Phys.Rev. D67 (2003) 034011, [hep-ph/0202008].

[15] M. Bleicher, F. Liu, A. Keranen, J. Aichelin, S. Bass, et al., Overpopulation of anti-Omega in $p$ p collisions: A Way to distinguish statistical hadronization from string dynamics, Phys.Rev.Lett. 88 (2002) 202501, [hep-ph/0111187].

[16] M. Hladik, H. J. Drescher, S. Ostapchenko, T. Pierog, and K. Werner, Self-consistency requirement in high-energy nuclear scattering, Phys. Rev. Lett. 86 (2001) 3506-3509, [hep-ph/ 0102194 ].

[17] K. Werner, Core-corona separation in ultra-relativistic heavy ion collisions, Phys.Rev.Lett. 98 (2007) 152301, [arXiv:0704.1270].

[18] Pierre Auger Collaboration, A. Aab et al., Muons in air showers at the Pierre Auger Observatory: Measurement of atmospheric production depth, Phys. Rev. D90 (2014), no. 1012012 , [arXiv: 1407.5919$]$. [Erratum: Phys. Rev.D92,019903(2015)].

[19] L. Cazon, R. A. Vazquez, A. A. Watson, and E. Zas, Time structure of muonic showers, Astropart. Phys. 21 (2004) 71-86, [astro-ph/0311223].

[20] L. Cazon, R. A. Vazquez, and E. Zas, Depth development of extensive air showers from muon time distributions, Astropart. Phys. 23 (2005) 393-409, [astro-ph/ 0412338 ].

[21] L. Cazon, R. Conceicao, M. Pimenta, and E. Santos, A model for the transport of muons in extensive air showers, Astropart.Phys. 36 (2012) 211-223, [arXiv: 1201.5294 ].

[22] Pierre Auger Collaboration Collaboration, P. Abreu et al., The Pierre Auger Observatory II: Studies of Cosmic Ray Composition and Hadronic Interaction models, Proceeding of the 32nd ICRC, Beijing, China (2011) [arXiv:1107.4804].

[23] ATLAS Collaboration Collaboration, G. Aad et al., Rapidity gap cross sections measured with the ATLAS detector in pp collisions at $\sqrt{s}=7$ TeV, Eur.Phys.J. C72 (2012) 1926, [arXiv:1201.2808].

[24] Particle Data Group Collaboration, C. Amsler et al., Review of particle physics, Phys. Lett. $\mathbf{6 6 6 7}$ (2008) 1.

[25] EHS-NA22 Collaboration, M. Adamus et al., Charged particle production in $k+p$, pi+ $p$ and $p p$ interactions at 250-gev/c, Z. Phys. C39 (1988) 311-329.

[26] https://web.ikp.kit.edu/corsika/ Collaboration, D. Heck, J. Knapp, J. Capdevielle, G. Schatz, and T. Thouw, Corsika: a monte carlo code to simulate extensive air showers, Wissenschaftliche Berichte, Forschungszentrum Karlsruhe FZKA 6019 (1998).

[27] Pierre Auger Collaboration, A. Aab et al., Depth of maximum of air-shower profiles at the Pierre Auger Observatory. I. Measurements at energies above $10^{17.8} \tilde{A} c \breve{A} L \tilde{A} \tilde{c} \breve{A} L^{\prime} e V$, Phys.Rev. D90 (2014), no. 12 122005, [arXiv:1409.4809].

[28] H.-J. Drescher, Remnant break-up and muon production in cosmic ray air showers, Phys. Rev. D77 (2007) 056003, [0712.1517]. 